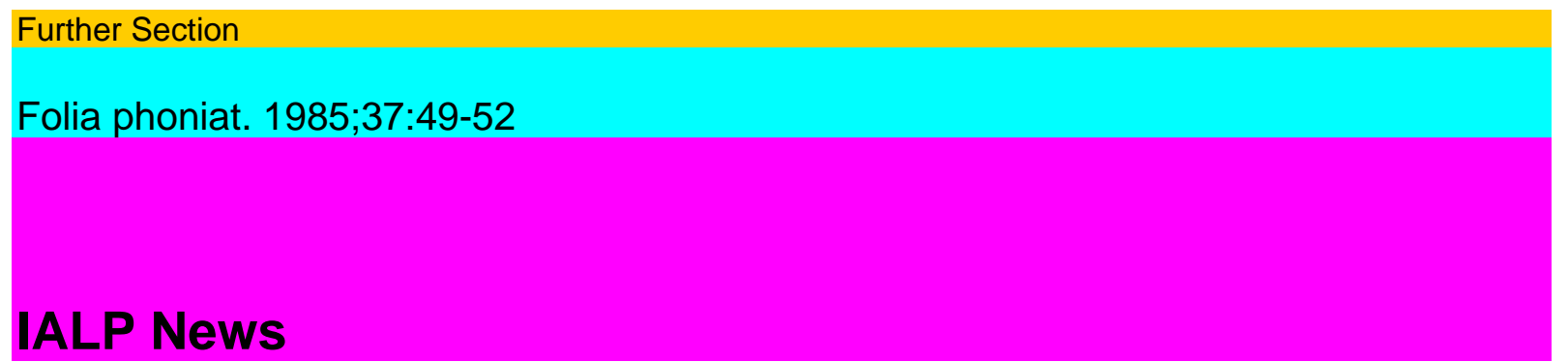

Informative and consultative status (category B) with $\quad$ UNESCO, UNICEF, WHO, ECOSOC and CIOMS

Prof. Lucio Croatto: 65 years!

Such an anniversary cannot be missed. IALP without Lucio is like a car without petrol. Born in Trieste on January 27th 1920, he graduated in Medicine at the University of Padova in 1945, specialized in ORL in 1949, became Libera Docente in Phoniatrics in 1956 and Associate Professor in Phoniatrics in 1982. With Prof. C. Tagliarini he set up in 1969 the first University School of Speech Therapy at Padova University and recently took pail in the new formation of Italian Phon-iatricians. He founded and still manages the Phoniatrica latina, rhista intemazionale di foniatria e logopedia.

Since 1953 Lucio Croatto has been one of the leading personalities of IALP, at first as Board member and as President from 1962 to 1968 . He was the organizer of the Xllth IALP Congress held in Padova in 1962.

Always enthusiastic for his teaching and for his research work, he still communicates to everyone a joy of living which makes him one of the most popular members of our association. Ad multos annos, caro Lucio!

Cairo: Symposium on Vocal Folds

A symposium on vocal fold histopathology, developmental and benign aspects, was organized by a Committee composed of Dr. W.-J. Gould, Dr. M. Hirano, Dr. Nasser Kotly and Dr. S. Vecerina. This symposium was sponsored by the Voice Foundation New York and was hosted by the Ain Shams University Cairo. It took place on March 5 and 6, 1984.

The organizing Committee has invited scientists who have performed special research in this field. The participants who took part in the symposium were: S. Kurita: Comparative histology of the vocal folds. W. Kersing: Muscles of the vocal folds in different age-groups. M. Hirano: Developmental histology of the vocal

folds: Growth and aging of the vocal folds. S. Fex and Bibifex: Small vocal fold abnormalities and

their influence on phonation. M. Frenzel: Ultrastructure and histochemistry of vocal folds and polyps. O. Kleinsasser: Clinicohistological aspects of benign lesions of the vocal folds.

B. Fritzell: Aspects of Reinker edema.

M. Nasser Kotby: Histopathology of the small associated benign lesions of the vocal folds.

J. Kirchner: Nonepithelial benign neoplasms of the larynx.

W. Lehmann: Granulomas of the larynx.

These presentations are now in print and will be published as the proceedings of this symposium in the

United States.

ler Séminaire International Phoniatrie et Logopédie Aujourd'hui

Ce séminaire s'est tenu à St-Vincent (Italie) du 15 au 18 octobre 1984 sous les auspices de $\Gamma$ IALP et de $\Gamma$ Union Européenne des Phoniatres. Excellemment organise par les Professeurs 
Rossi et Schindler de $\Gamma$ Uni-versité de Turin, il a fait le point de nos connaissances actuelles dans tous les domaines touchant à la communication humaine: linguistique, psychologie, dyspho-nies, dyslalies mécaniques périphériques, bégaiement, aphasie, dysarthrie, retard de langage, surdité, audimu-tisme. Les orateurs avaient été conviés de tous les pays d'Europe et même des EtatsUnis et de $\Gamma$ Argentine. Les très nombreux participants ont apprécié la qualité des exposes donnés en italien, français et anglais.

Ces travaux ont été groupés en un gros volume (à paraître en italien seulement) publié aux Edizioni Omega, Vie Passalacqua 19, 10122 Torino; prix de vente: 35,000 L.

Union of European Phoniatricians

The XПth Congress of the Union of European Phoniatricians will be held in Poznaí (Poland), September 12-15, 1985. Programme of the Congress: (1) Phoniatrie aspects in neurologic and psychiatric diagnosis (main topic), (2) The present state and developmental prospects of child audiology (postgraduate course).

Preliminary registration forms to be sent in by December 31, 1984. President of the Organizing Committee: Prof. A. Pruszewicz, MD, Department of Phoniatrics and Audiology, K. Marcinkowski University School of Medicine, ul. Przybyszewskiego 49, 60-355 Poznań (Poland).

50

IALP News

Book Reviews $\cdot$ Buchbesprechungen $\cdot$ Livres nouveaux

P. Ptacek, P. Kricos, M. Hyman, J. Black Index to Speech, Language and Hearing Journal Titles 1954-78

Houston 1979; 305 pp.

Toute investigation et tout progrès doivent se baser sur les travaux antérieurs realises dans le champ determine de $\Gamma$ étude. Chercher et trouver la littérature scientifique adequate est frequemment très difficile. C'est ainsi qu'un laboratoire d'investigation affirme qu'il est plus économique de recommencer une experience que de trouver ce qui a été écrit à ce sujet. II est exact que Tampleur de $\Gamma$ investigation augmente chaque jour: le nombre des revues augmente, la quantité de lecteurs qui cherchent du materiel se mul-tiplie. En outre, ces lecteurs désirent trouver ce materiel recent rapidement.

Pour faciliter cette recherche, les auteurs de ce livre resument ce qui a été publié sur Гaudition et le langage dans 31 revues depuis 1954 jusqu'à 1978. A notre surprise, ces revues sont toutes d'Amérique du Nord, sauf Folia phoniatrica.

II est intéressant d'observer la classification que les auteurs font de toutes les connaissances en dix categories, subdivisées à la fois en 100 subcatégories. Cette classification de la matière pourrait être utile et s'ap-pliquer aux revues, bibliothèques, livres, etc...

J. Perelló

A. Girolami-Boulinier

Les niveaux actuels dans la pratique du

langage oral et écrit

Collection d'Orthophonie Masson, Paris 1984254 pp.

L'auteur, dont $\Gamma$ expérience en pédagogie et en reeducation est grande, nous apporte les resultats de nombre d'années de travail. Les rapports entre langage, lecture et orthographie sont disséqués. Le langage oral et écrit est étudié en comprehension et en expression; il est en outre compare avec les moyennes que donnent les tests de reference effectues aux divers ages scolaires. Les fautes phonétiques ou linguistiques montrent la tendance vers les différents types de dyslexic 
L'auteur examine fort justement la nature des fautes constatées en lecture orale et la comprehension du texte en lecture silencieuse. Les sequelles de retard de parole et les troubles perceptifs du langage oral et de la lecture se traduisent ensuite dans le langage écrit. L'analyse des difficultés rencontrées donne la possibi-lité d'apporter un remède efficace aux deficits individuels constates. Cet ouvrage très bien documenté sera de grande utilité pour tous les pedagogues, logopédis-tes et médecins s'occupant des troubles de la communication.

\section{A. Muller}

H.H. Wängler, J. Baumann- Wängler Phonetische Logopädie

Marhold, Berlin 198495 pp.; DM 37.-

It is the second issue from page 97 to 192 of a volume on logopedy that will have 1,248 pages.

As it can be imagined this work will be of an extraordinary importance in logopedy. In this issue the articulation of the phoneme /S/, its troubles, diagnosis and therapy are widely studied.

J. Perelló

Paul Eling

Studies on Laterality

These No. 0983, Nimègue $128 \mathrm{pp}$.

Le volume nous offre une etude complete de $\Gamma$ état actuel des connaissances sur la latéralité, non dans le sens traditionnel, mais comme fonctionnement cerebral à travers le langage. On décrit le système fonction-nel qui n'est pas précisément le classique centre cerebral, décrit dans tous les traités de Neurologie classique.

\section{IALP News}

51

Dans certains chapitres, Tauteur expose ses experiences sur Taudition binaurale et des faits nouveaux pouvant expliquer certaines predominances auditives; les procédés pour $\Gamma$ exploration d'une latéralité déter-minée ne peuvent se généraliser à d'autres zones.

L'auteur se demande dans quelle mesure les mé-canismes qui produisent les differences droitegauche sont indépendants et quelle est la nature du facteur commun sous-jacent aux signaux de latéralité et pour-quoi les deux hemispheres, identiques anatomique-ment, ont des fonctions différentes. II semblerait que chez les enfants, les deux hemispheres soient équipo-tents et que la latéralité ne s'établisse qu'à la puberté. L'auteur s'étonne du nombre réduit d'études réalisées sur la véracité et la validité des différentes méthodes mises en æuvre pour examiner et mesurer la latéralité, surtout de certaines qui ne sont pas naturelles. Malgré le manque de confiance en certaines explorations, il est surprenant de voir leurs résultats acceptés et inter-prétés sans doutes ni hesitations. Ce livre contient en plus, un grand nombre de conceptions et de faits origi-naux qui en rendent nécessaire la lecture à tous ceux qui s'intéressent au phénomène de la latéralisation.

\section{J. Perelló}

Eva Bernoulli

Mein Weg zu Sprache und Stimme

Friedrich Reinhardt Verlag, Basel 1984192 pp.; SFr. 19.80 ISBN 3-7245-0547-7

Eva Bernoullis Lebenserinnerungen sind ein Stuck Basler Kulturgeschichte und ein persönlicher Bericht über die Pionierzeit der Logopädie, vor allem in der Schweiz. Eva Bernoulli, Ehrenmitglied der Schweize-rischen Gesellschaft für Phoniatrie, Logopädie und Audiologie, schildert ihr bewegtes Leben, dem auch Enttäuschungen und Kämpfe nicht erspart blieben, in 
bescheidener, doch ansprechend humorvoll-heiterer Art. Die Lektüre ihres Buches vermag daher alle, die mit Sprache und Stimme als Künstler, Therapeuten oder Ärzte zu tun haben, aber auch jeden Basler, der am Kulturleben seiner Stadt interessiert ist, zu fes-seln.

Eberhard Kaiser

William H. Perkins (ed.) Hearing Disorders

Thieme, Stuttgart 1984

170 pp., 1 fig., 10 tab.; DM 58.-

ISBN 3-13-643901-5 (TSI)

This is another volume of the series on the treatment of communication disorders, written by 21 authors on themes in which they are authorities.

Indeed, most of the chapters are contributions, from different angles, to the education of deaf children. Nevertheless, there are chapters devoted to the study of the auditory problems in adults and old people.

The diversity of the authors and their particular points of view do not allow conclusions to be drawn on the recommended methodology for the education of deaf children.

Each chapter ends with a short bibliography all in English.

J. Perelló

E. Wilken

Sprachförderung bei Kindern mit

Down-Syndrom

Marhold, Berlin 1979; 3e éd. 148 pp., 3 fig.; DM 20.-

Cet excellent livre, consacré à $\Gamma$ étude et au traite-ment des difficultés de langage chez l'enfant mongo-lien, a le mérite d'en être à sa $3 \mathrm{e}$ edition mise à jour et augmentée de divers nouveaux themes.

L'auteur justifie le nom du syndrome, en expose Гétiologie et ses caractéristiques. II passe ensuite au theme fundamental du livre: le langage de ces enfants, son développement et ses défauts. II examine minu-tieusement les anomalies de la bouche, du larynx, du nez, de la respiration, de $\Gamma$ audition, de $\Gamma$ articulation et de la phonation.

Une grande part est réservée à la thérapeutique du retard linguistique. L'auteur expose les exercices de respiration, de mobilité buccale, la stimulation auditive et le développement du langage.

Ce petit livre, destine exclusivement à la logopédie de l'enfant affecté du syndrome de Down, remplit par-faitement sa fonction.

J. Perelló

52

IALP News

Giovanna Bruno

Physiopathology of Verbal Communication

Verduci, Roma 1984284 pp., 57 fig. ISBN 88-7620-048-7

This volume was meant to be a study text for physicians who want to specialize in phoniatry, the official teaching of which has begun in some Italian universities.

The volume has the structure of a teaching book with equilibrium and hierarchical arrangement of 
themes, expositive clarity, demonstrative illustration, and so on. It treats only the troubles of language and will be followed by another volume of the pathology of the spoken and singing language. J. Perelló 